\title{
A High-Pressure Gaseous-Argon TPC (HPgTPC) as a Component of the DUNE Near Detector
}

Tanaz Angelina Mohayai, for the DUNE Collaboration CPAD Instrumentation Frontier Workshop March 19, 2021 


\section{Outline}

\section{-DUNE}

$\star^{\text {Primary Goal }}$

$\star$ Role of a High Pressure Gas-Ar TPC (HPgTPC)

-HPgTPC as a Component of a Near Detector:

$\star$ Conceptual Design

$\star$ Expected Performance

$\star$ R\&D Efforts

-Summary 


\section{Deep Underground Neutrino Experiment (DUNE)}

Primary goal of DUNE is to reduce the uncertainties in the oscillation measurements to a few \% level

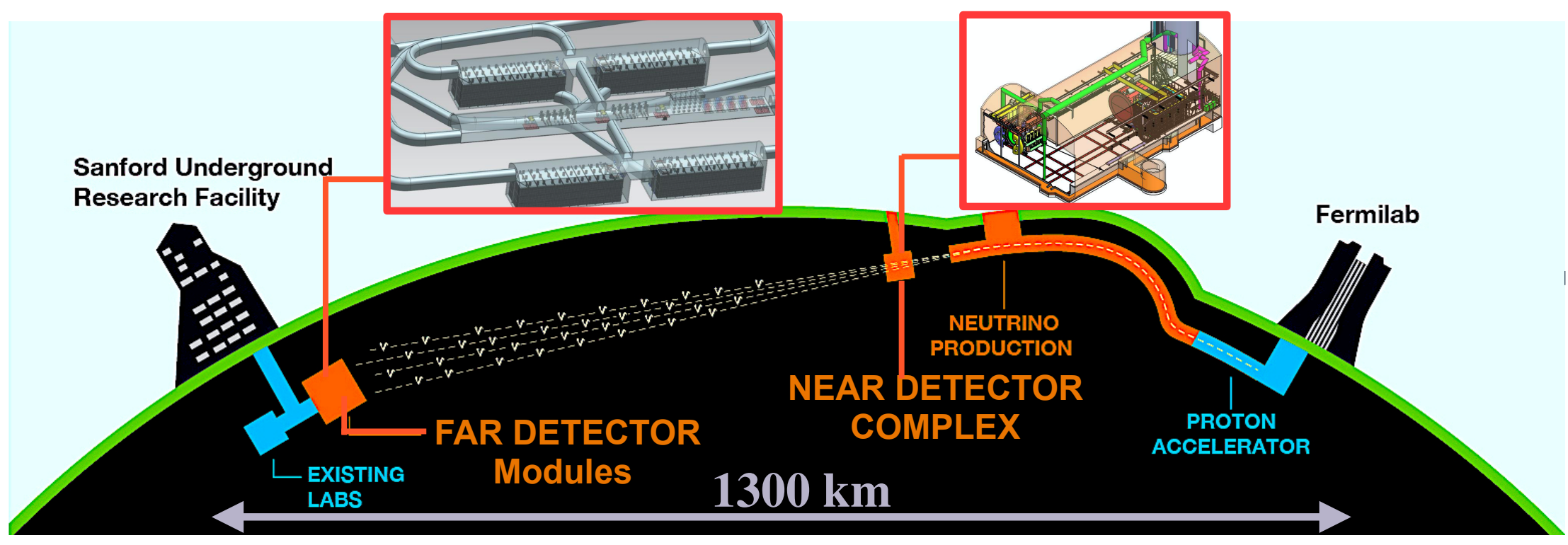

- The observable is the ratio of appearance events in the liquid Argon time projection chamber far detector (FD) modules to suite of near detectors (ND):

oscillation probability

$$
\frac{N_{\nu_{e}}^{F D}\left(E_{\text {reсo }}\right)}{N_{\nu_{\mu}}^{N D}\left(E_{\text {reco }}\right)}=\frac{\left.\int P_{\nu_{\mu} \rightarrow \nu_{e}}\left(E_{\nu}\right) \times \Phi_{\nu_{e}}\left(E_{\nu}\right) \times \sigma_{\nu_{e}}\left(E_{\nu}\right)\right] \times \epsilon_{\nu_{e}}^{F D}\left(E_{\nu}\right) \times\left[S_{\nu_{e}}^{F D}\left(E_{\nu} \rightarrow E_{\text {reco }}\right)\right] d E_{\nu}}{\int\left[\Phi_{\nu_{\mu}}\left(E_{\nu}\right) \times \sigma_{\nu_{\mu}}\left(E_{\nu}\right) \times \epsilon_{\nu_{\mu}}^{N D}\left(E_{\nu}\right) \times S_{\nu_{\mu}}^{N D}\left(E_{\nu} \rightarrow E_{\text {reco }}\right)\right] d E_{\nu}}
$$

- Near detector should constrain uncertainties in near to far extrapolation as well as the uncertainties in the flux $(\Phi)$, cross section $(\sigma)$ and $v$-energy (migration matrix $S$ ) measurements and be a highly efficient detector $(\boldsymbol{\epsilon})$ 


\section{Dominant Sources of Uncertainty}

- Dominant sources of uncertainties are in cross sections/neutrino interaction models

- Nucleus is a complicated environment:

$\star$ Initial state of nucleons, nuclear effects, and final state interactions not yet fully understood and modeled

$\star$ Makes it difficult to infer the initial $v$-interaction and $v$-energy from final state topology, especially in heavier target nuclei

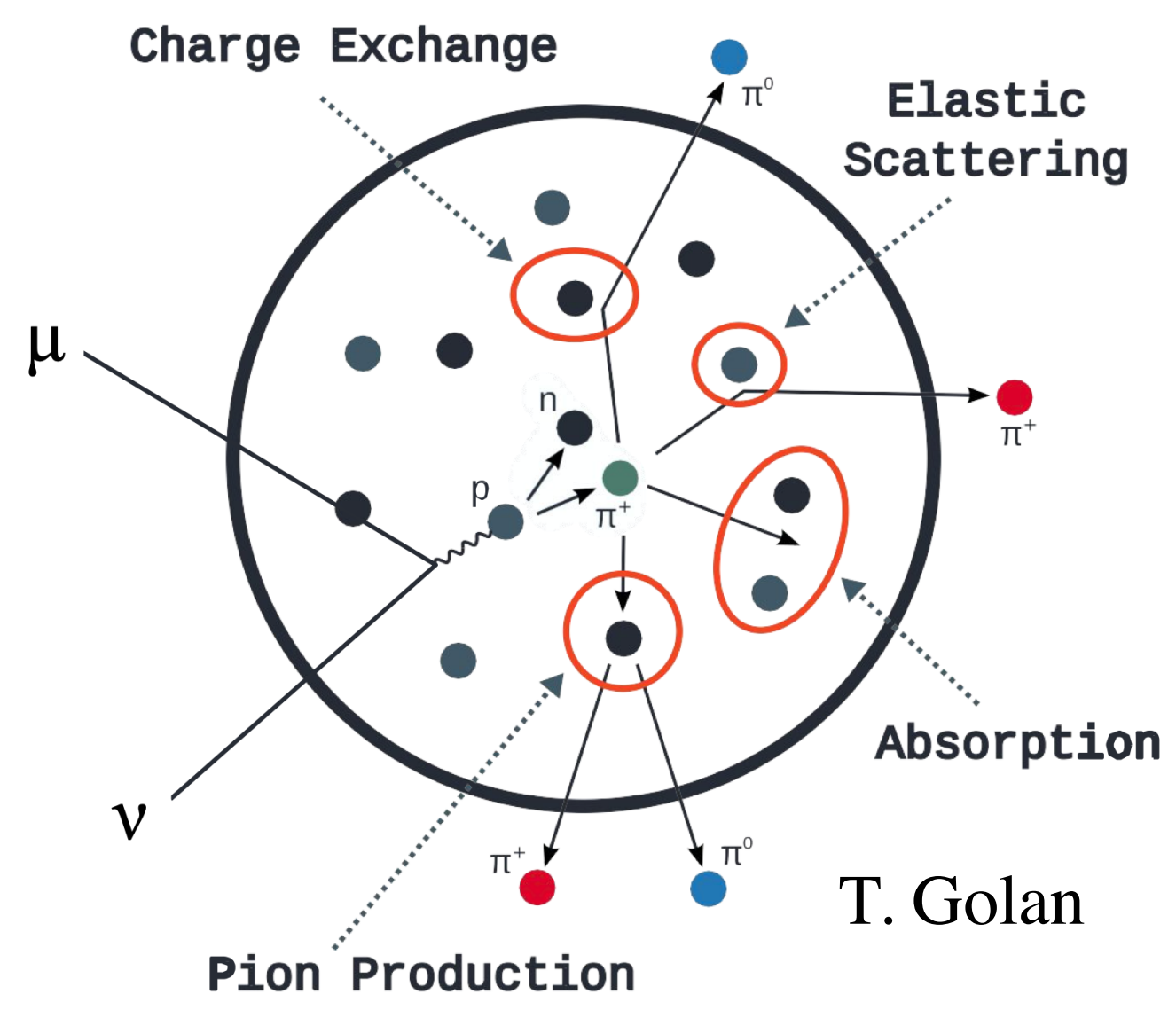




\section{Role of a High Pressure Gas TPC}

- HPgTPC can help constrain $v$-interaction and cross section uncertainties: $\star$ Its lower density $\left(\rho_{\mathrm{LAr}} / \rho_{\mathrm{GAr}} \approx 85\right.$ for $\left.10 \mathrm{~atm} \mathrm{GAr}\right)$ hence lower detection threshold makes it highly sensitive to lower energy charged particles that may not be seen in LAr

$\star$ Reveal discrepancies between different neutrino event generators at lower energies $\&$ get closer at choosing a more accurate $v$-interaction model as defined by our event generators GENIE, NEUT, \& NUWRO
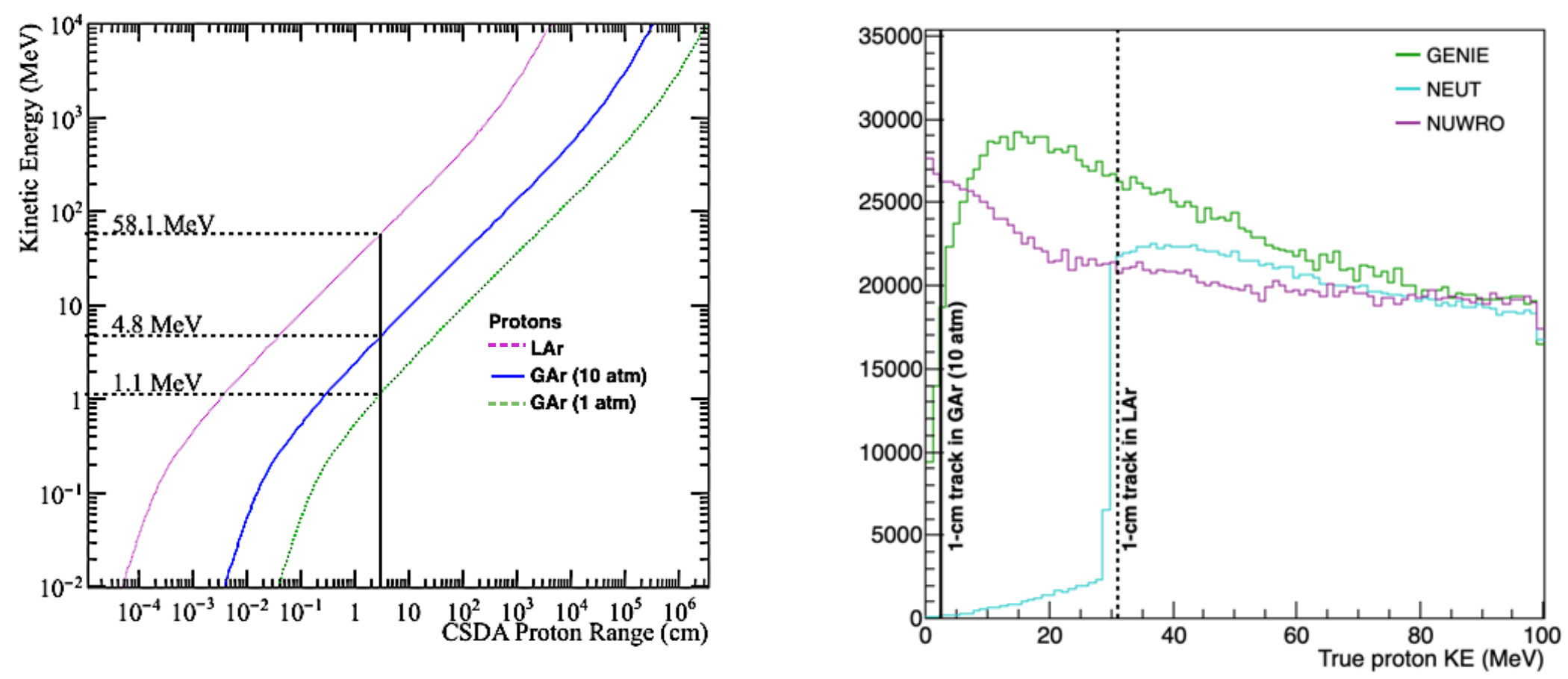


\section{DUNE Near Detector Complex}

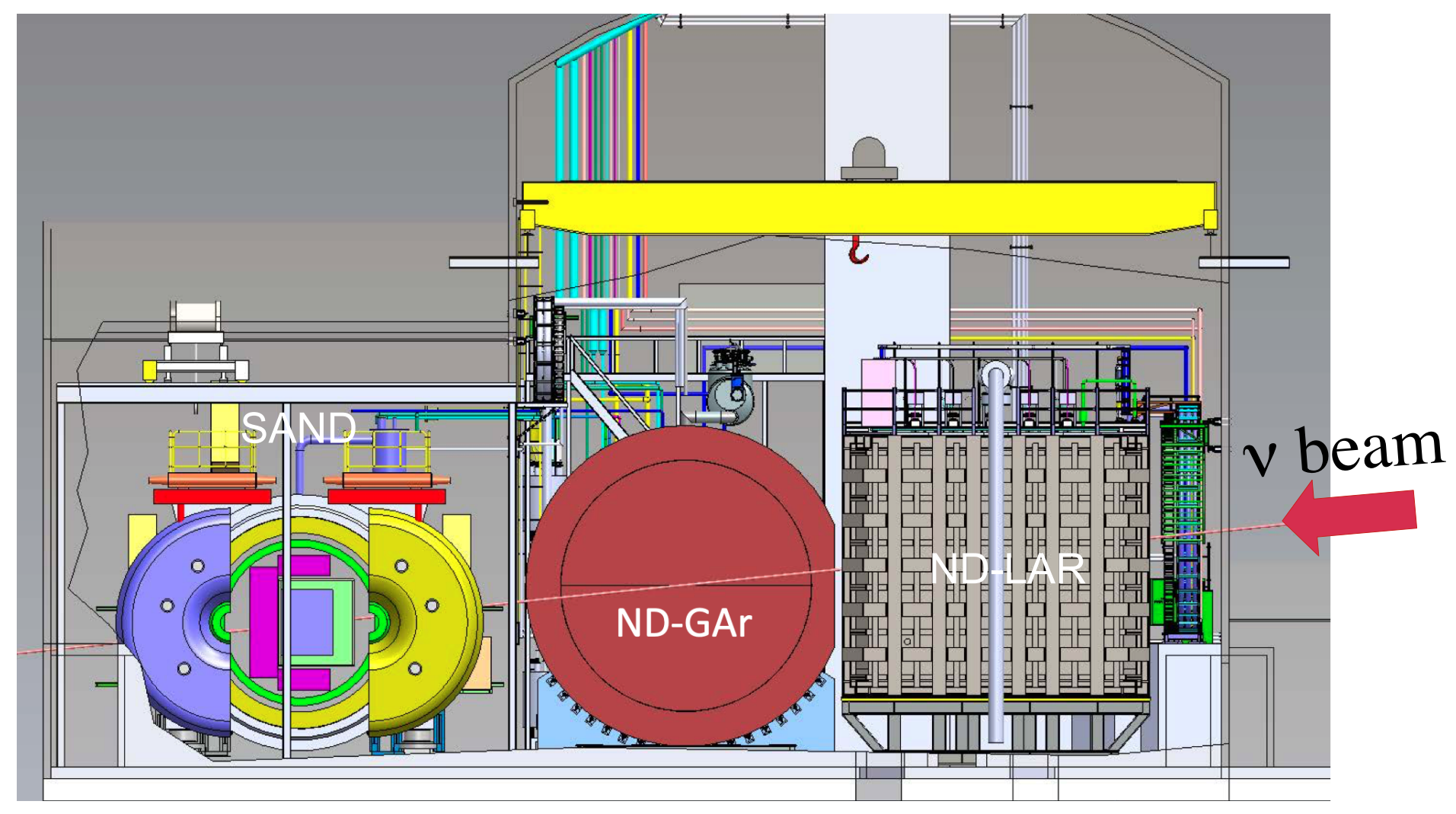

- Near detector hall houses various near detector components:

$\star$ ND-LAr, Liquid Argon time projection chamber

$\star$ ND-GAr, magnetized high pressure gaseous Argon time projection chamber (HPgTPC) surrounded by ECAL calorimeter

$\star$ SAND, system for on-axis neutrino detection (ND-LAr and ND-Gar move off-axis as part of the DUNE PRISM program) 


\section{ND-GAr Design}

- ND-GAr design:

$\star$ High Pressure Gas Argon TPC (HPgTPC):

- Will re-use ALICE's readout chambers

- Reference design $\mathrm{Ar}-\mathrm{CH}_{4}$ 90-10 gas mixture (97\% Ar interactions) at $10 \mathrm{~atm}$ $\star$ HPgTPC will be surrounded by ECAL calorimeter and superconducting magnet

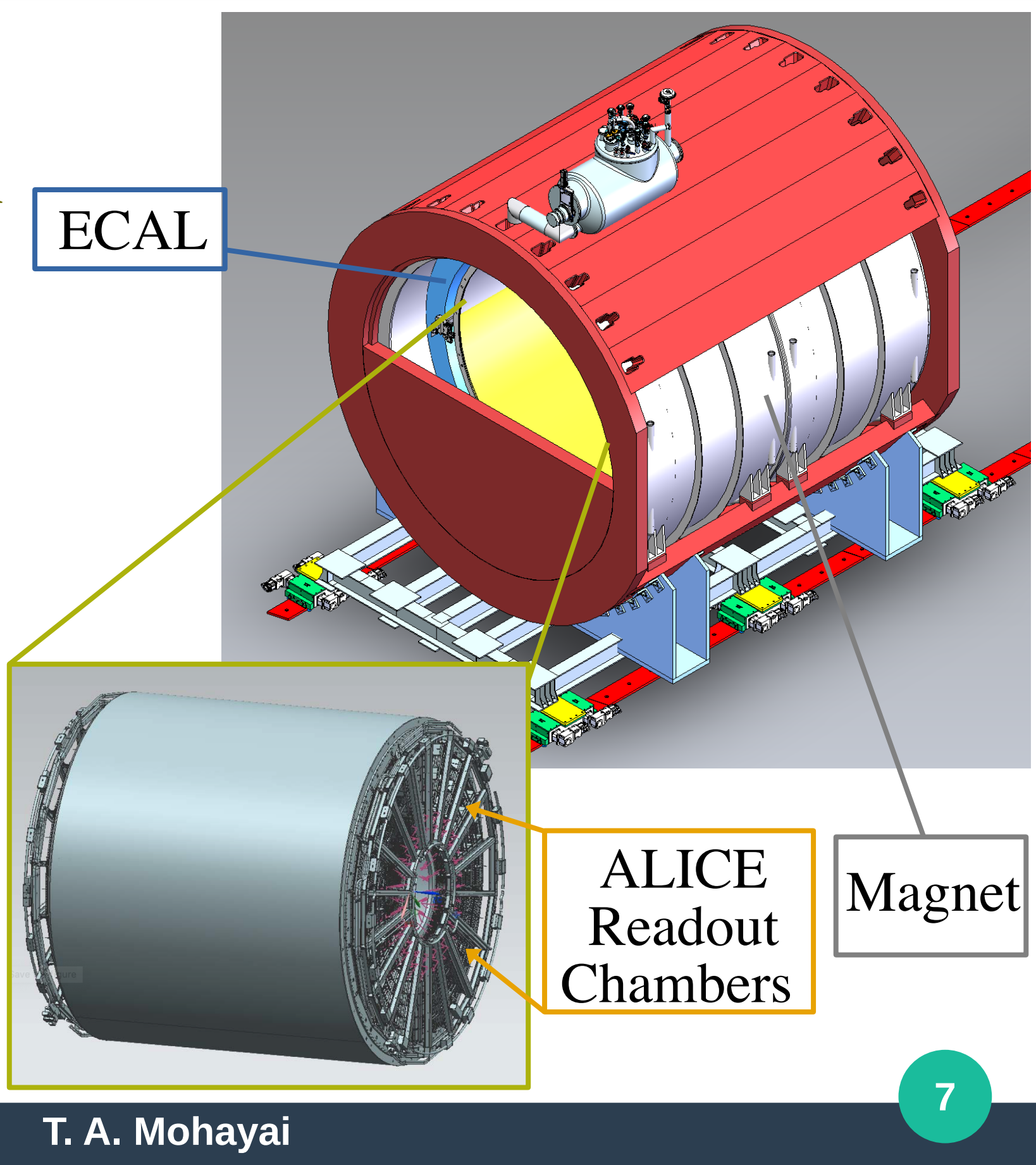




\section{HPgTPC Expected Performance}

- TPC the size of ALICE when pressurized (pressure comparable to PEP-4) can collect $2 \mathrm{M} v_{\mu} \mathrm{CC}$ events/ton of ${ }^{40} \mathrm{Ar} / \mathrm{year}$ and reach the DUNE physics goals

- Performance comparable to ALICE and PEP-4:

$\star$ Example: momentum resolution of $2.7 \%$ with latest HPgTPC reconstruction and excellent PID with $\mathrm{dE} / \mathrm{dx}$ resolution comparable to PEP-4

DUNE Simulation

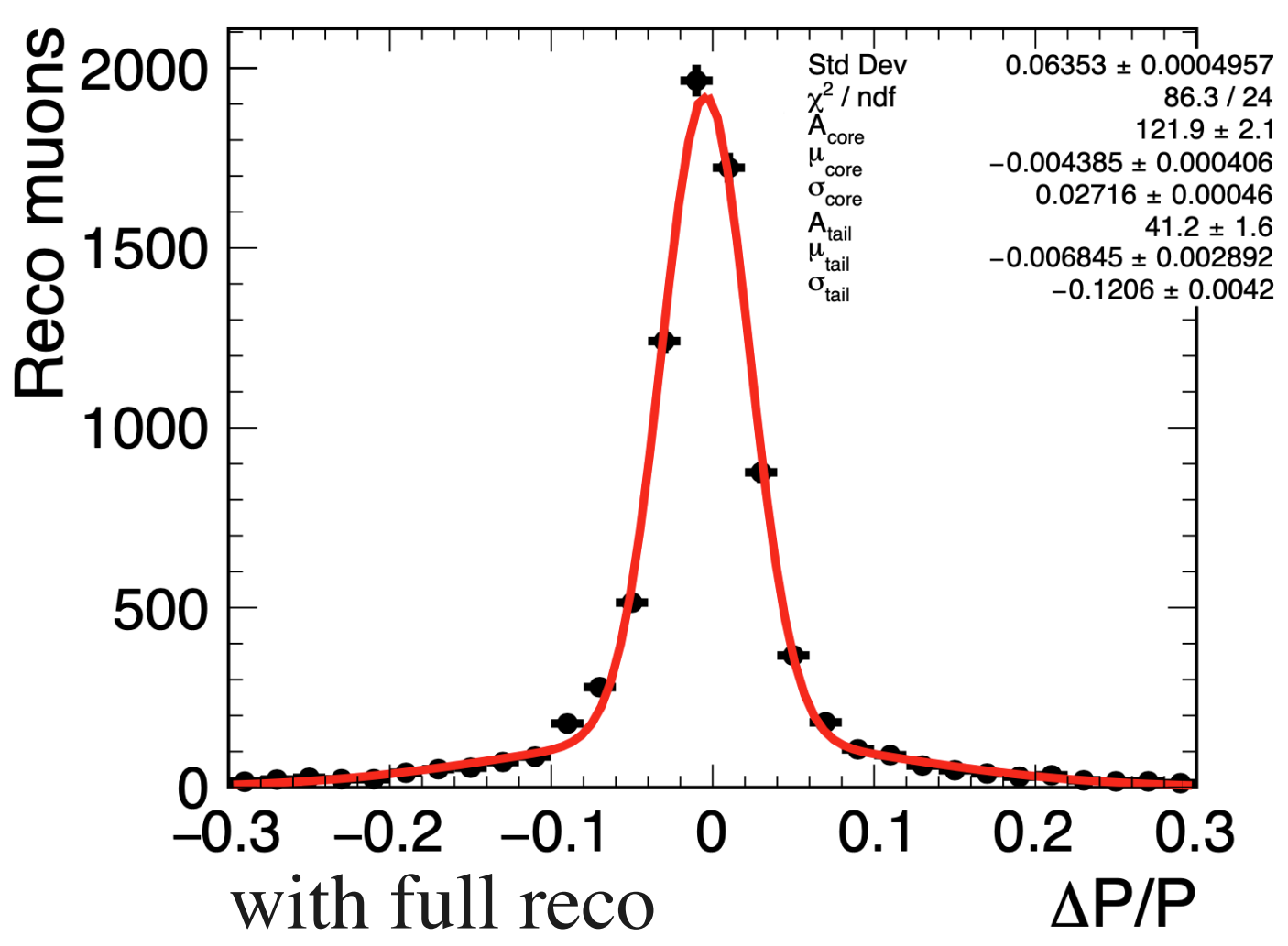

PEP4, $\mathrm{Ar}-\mathrm{CH}_{4}=80: 20 @ 8.5 \mathrm{~atm}$

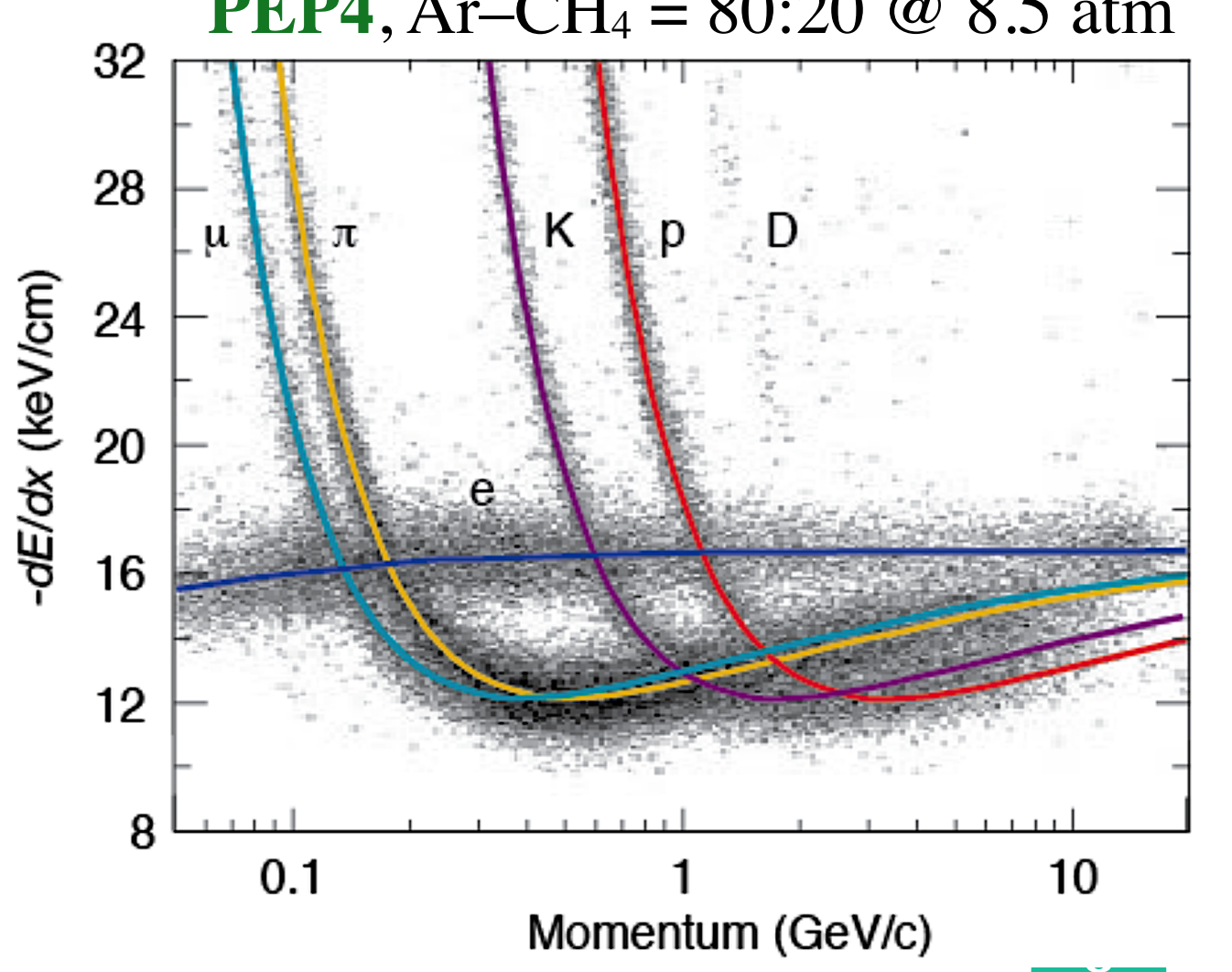




\section{HPgTPC Expected Performance}

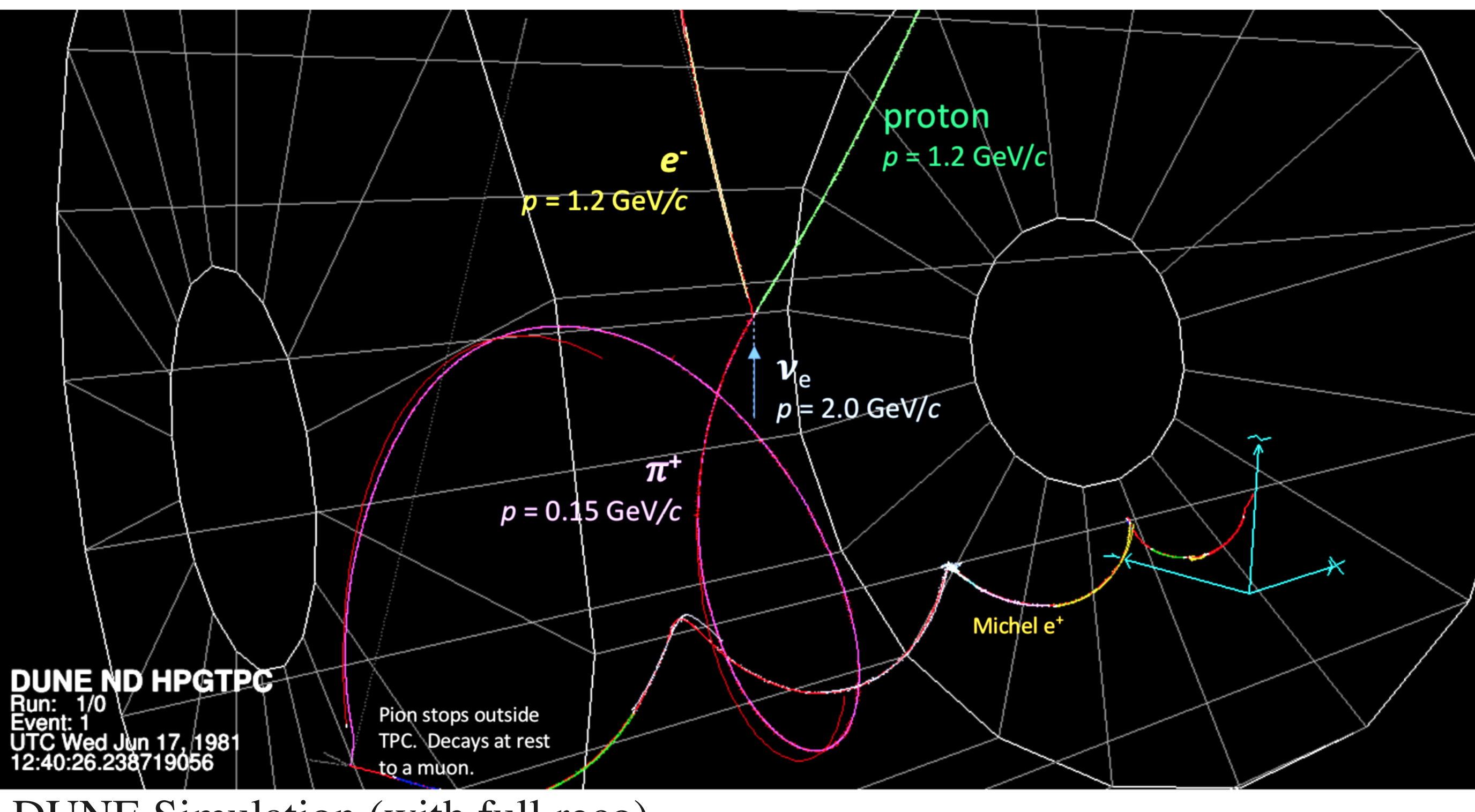

\section{DUNE Simulation (with full reco)}




\section{R\&D Efforts}
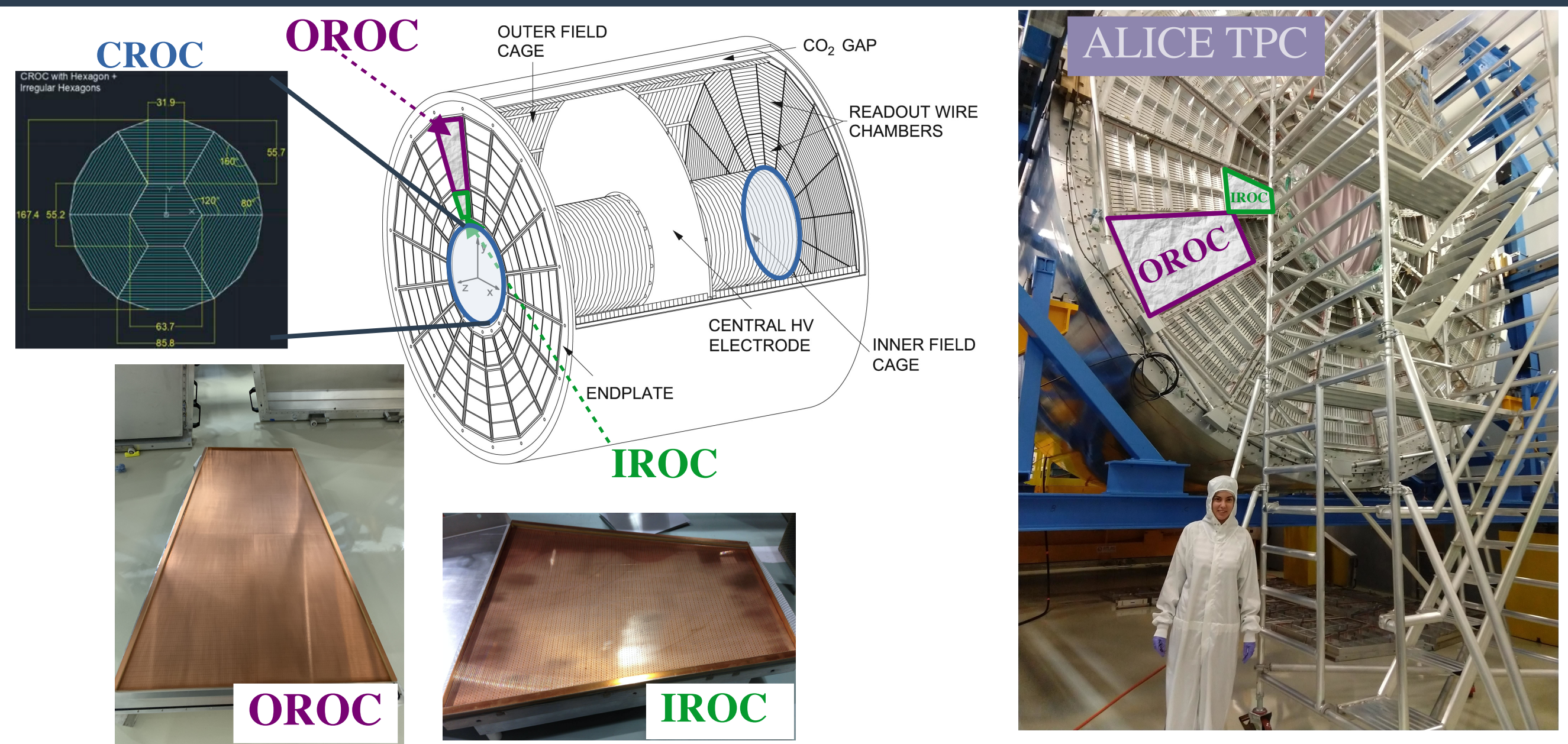

\section{OROC}

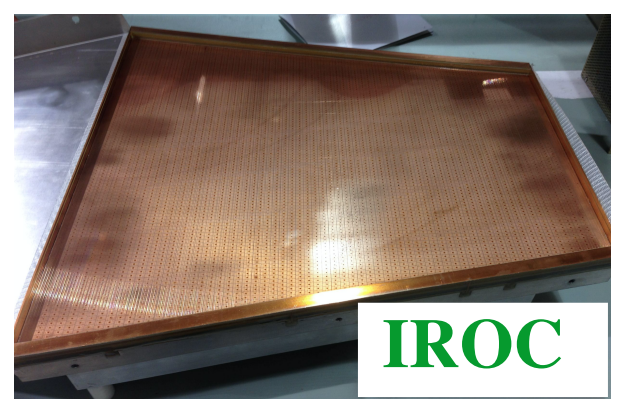

- Some of the required R\&D on the acquired ALICE inner and outer readout chambers:

$\star$ Test them @ various pressure points up to 10 atm - they operated at $1 \mathrm{~atm}$ in ALICE $\star$ Define a base gas mixture for them - $\mathrm{Ar}^{-\mathrm{CH}_{4}}$ (97\% of interactions on Ar), other gas mixtures also under investigation for their light properties and operational stability - There is also R\&D on building the central readout chambers (CROCs) 


\section{R\&D Efforts}

- Some of the criteria for choosing the right gas mixture and operating at high pressure:

$\star$ High drift velocity to control pile up

$\star$ Low diffusion for reasonable spatial resolution

$\star$ Strict purity requirements e.g. minimized $\mathrm{O}_{2}$ and $\mathrm{H}_{2} \mathrm{O}$ to prevent electron attachment $\star$ High voltage supplied to amplification/anode wires to account for reduced gain at pressure $>1 \mathrm{~atm}$ (can also optimize the gas mixture)
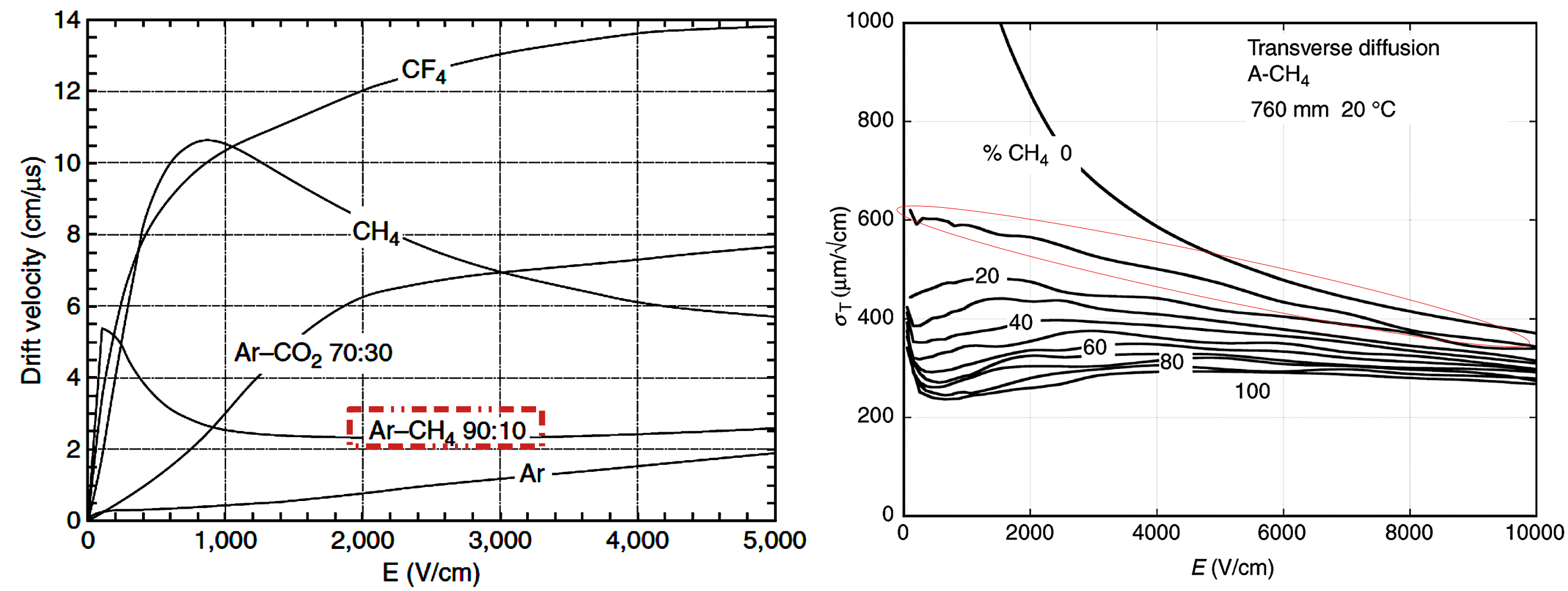

Sauli, F. “Gaseous Radiation Detectors: Fundamentals and Applications,” Cambridge: Cambridge University Press. doi:10.1017/CBO9781107337701.006 


\section{IROC Test Stand (GOAT) @ Fermilab}

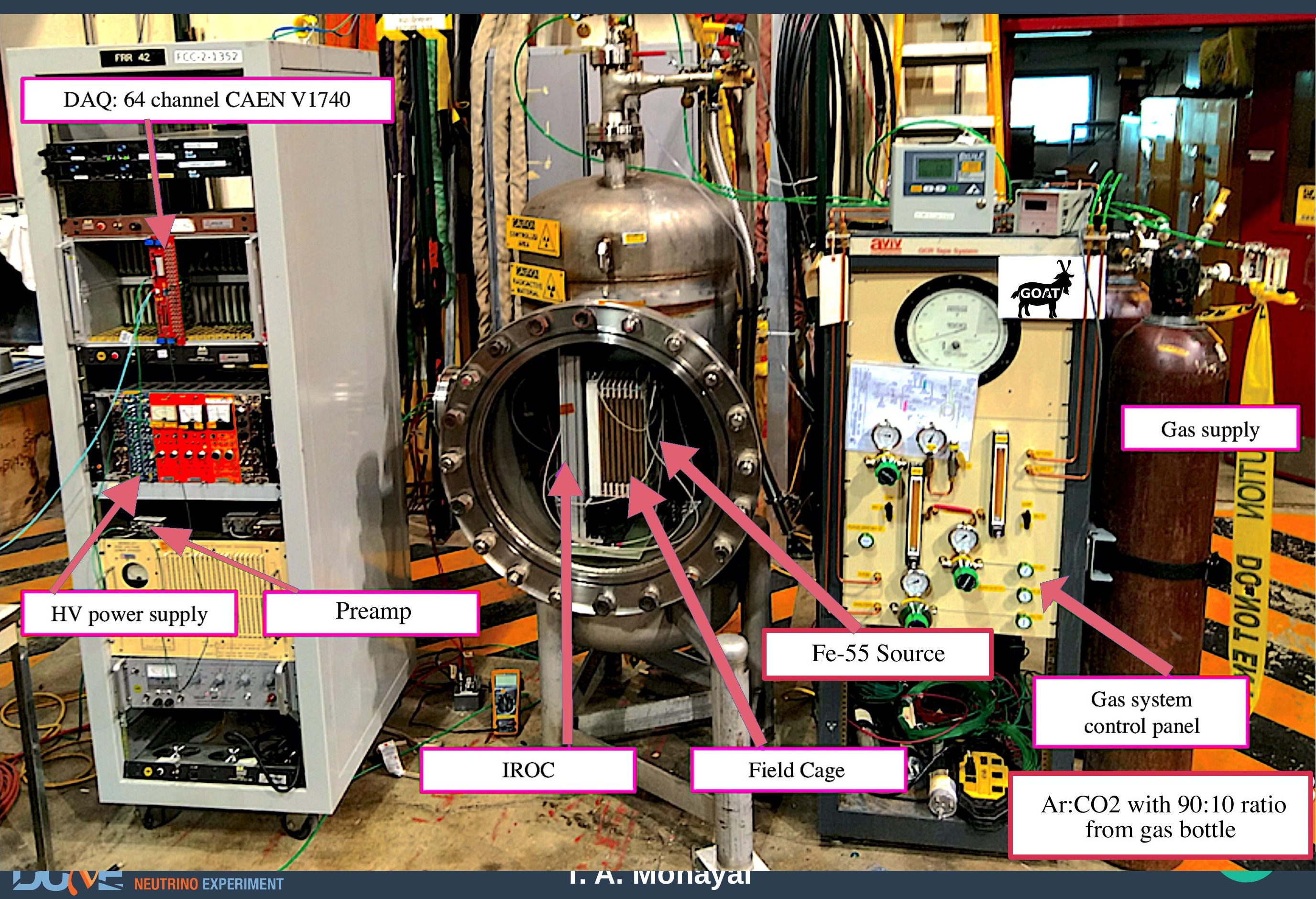




\section{The Signal}

Example single trigger waveform Anode: $1400 \mathrm{~V}$, pressure: $1 \mathrm{~atm}$

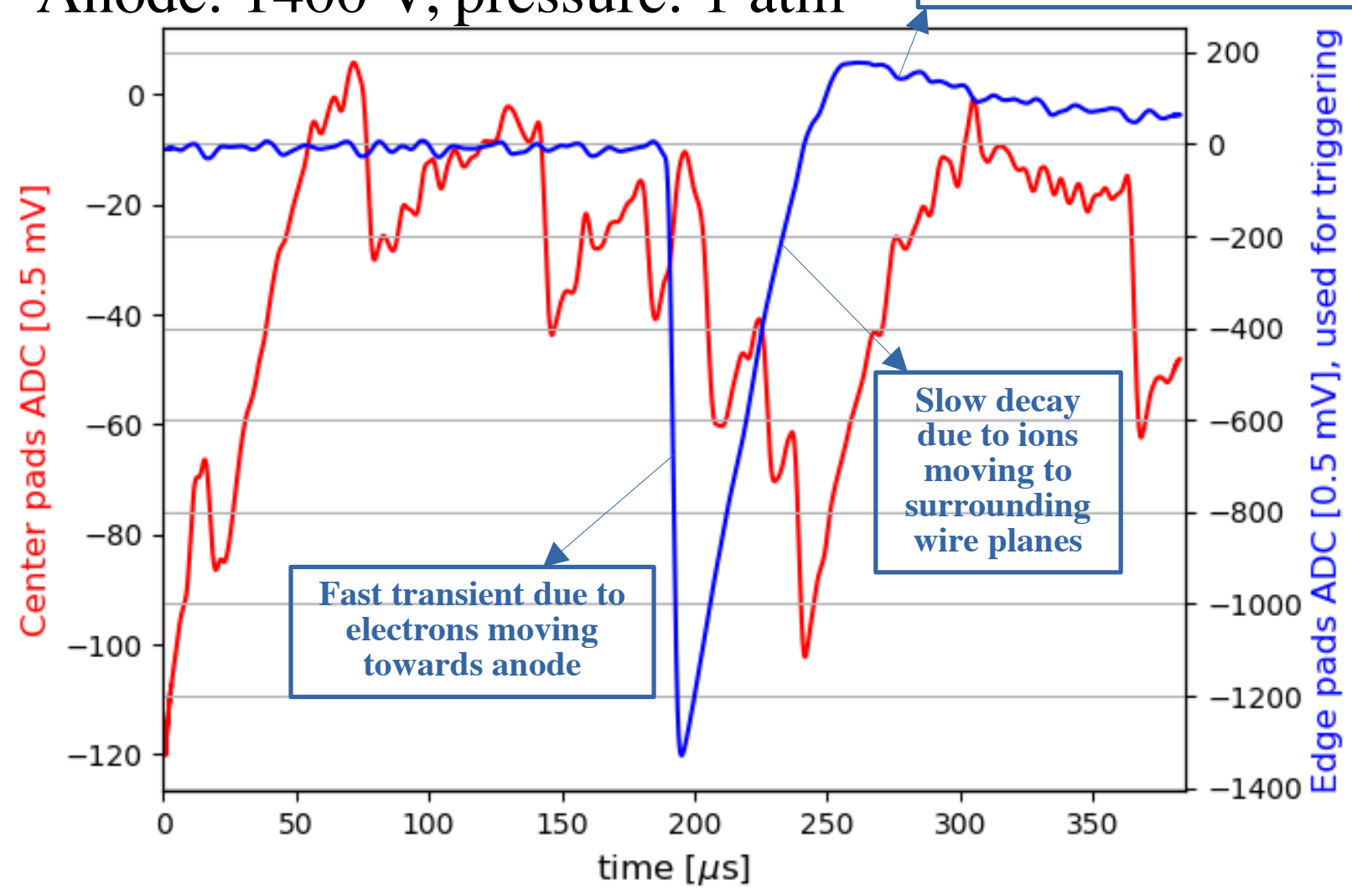

Long tail due to ions drifting back into the field cage volume

**note: charge sensitive inverting preamps used - signal shape slightly affected

- "Edge pads" (blue) only readout cosmics (solid angle of the source only limited to "center pads") - used for rejecting cosmic backgrounds

- Each peak in "center pad" waveform in red (with amplitude > peak-to-peak noise level) is an Fe-55 x-ray conversion 


\section{Gain at $1 \mathrm{~atm} \mathrm{Ar}-\mathrm{CO}_{2}$ 90:10}

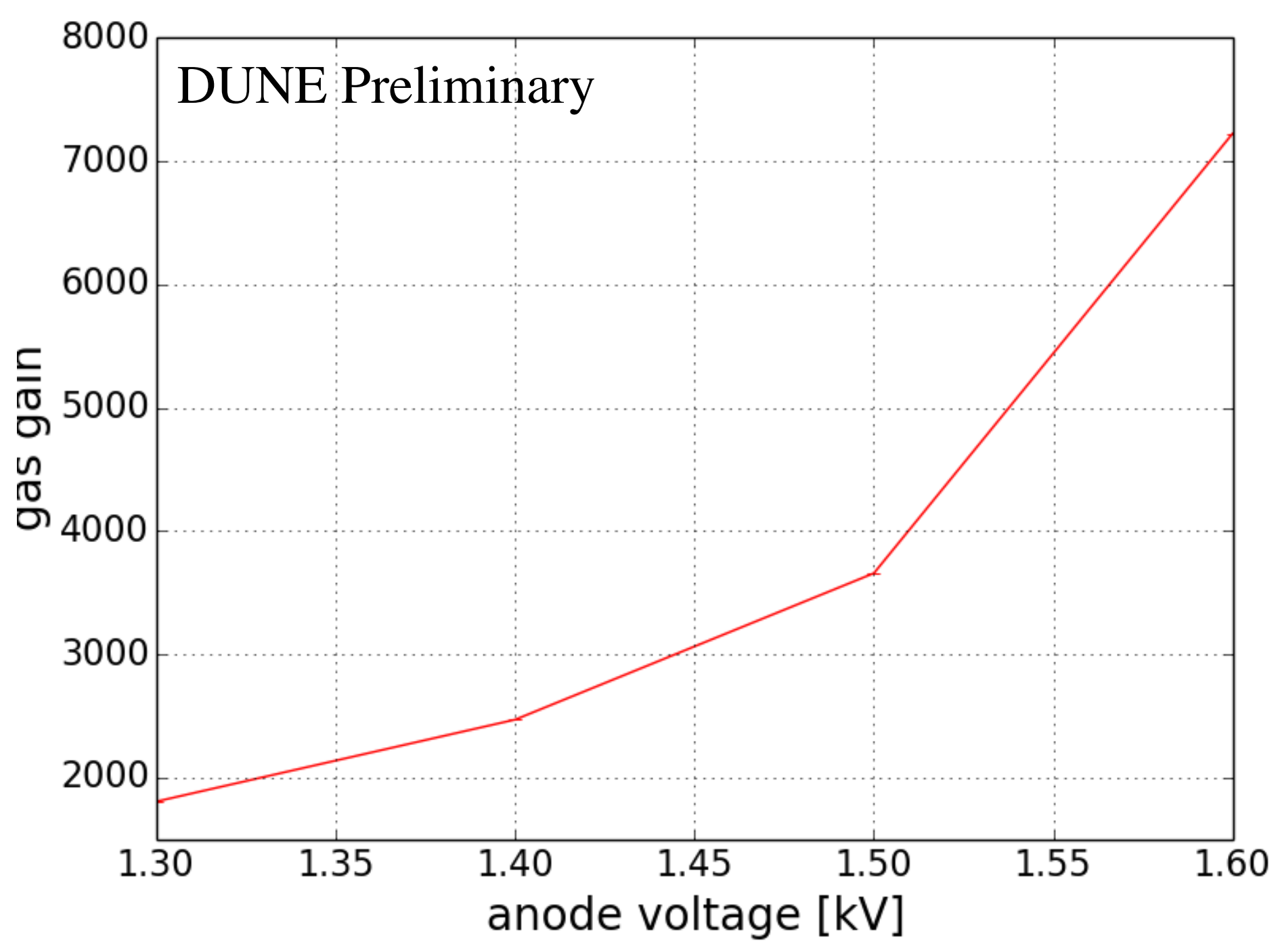

- The expected trend is present: for fixed pressure, a higher anode voltage results in higher gain 


\section{OROC Test Stand @ Royal Holloway University of London}

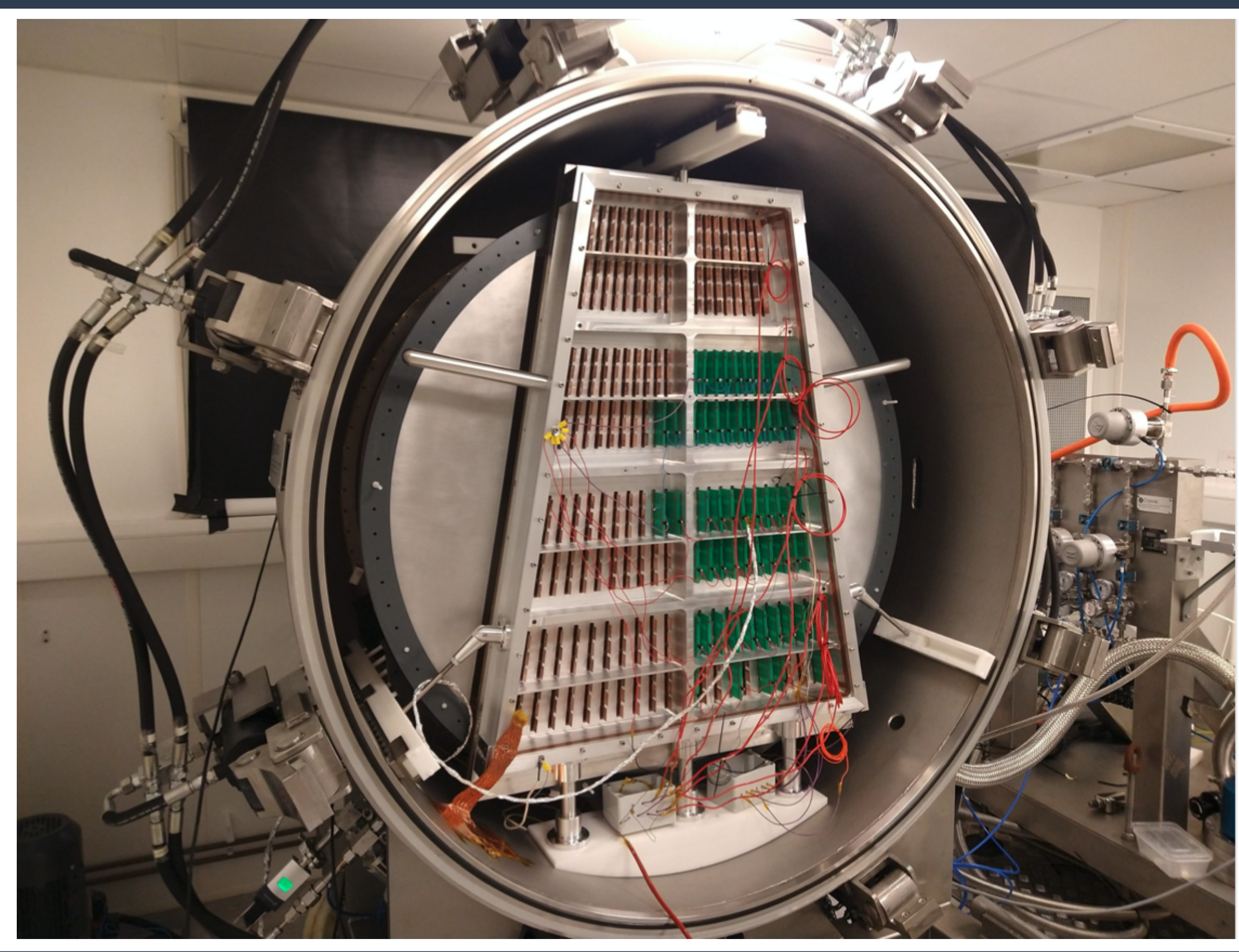




\section{The Signal}

Anode $1725 \mathrm{~V}$

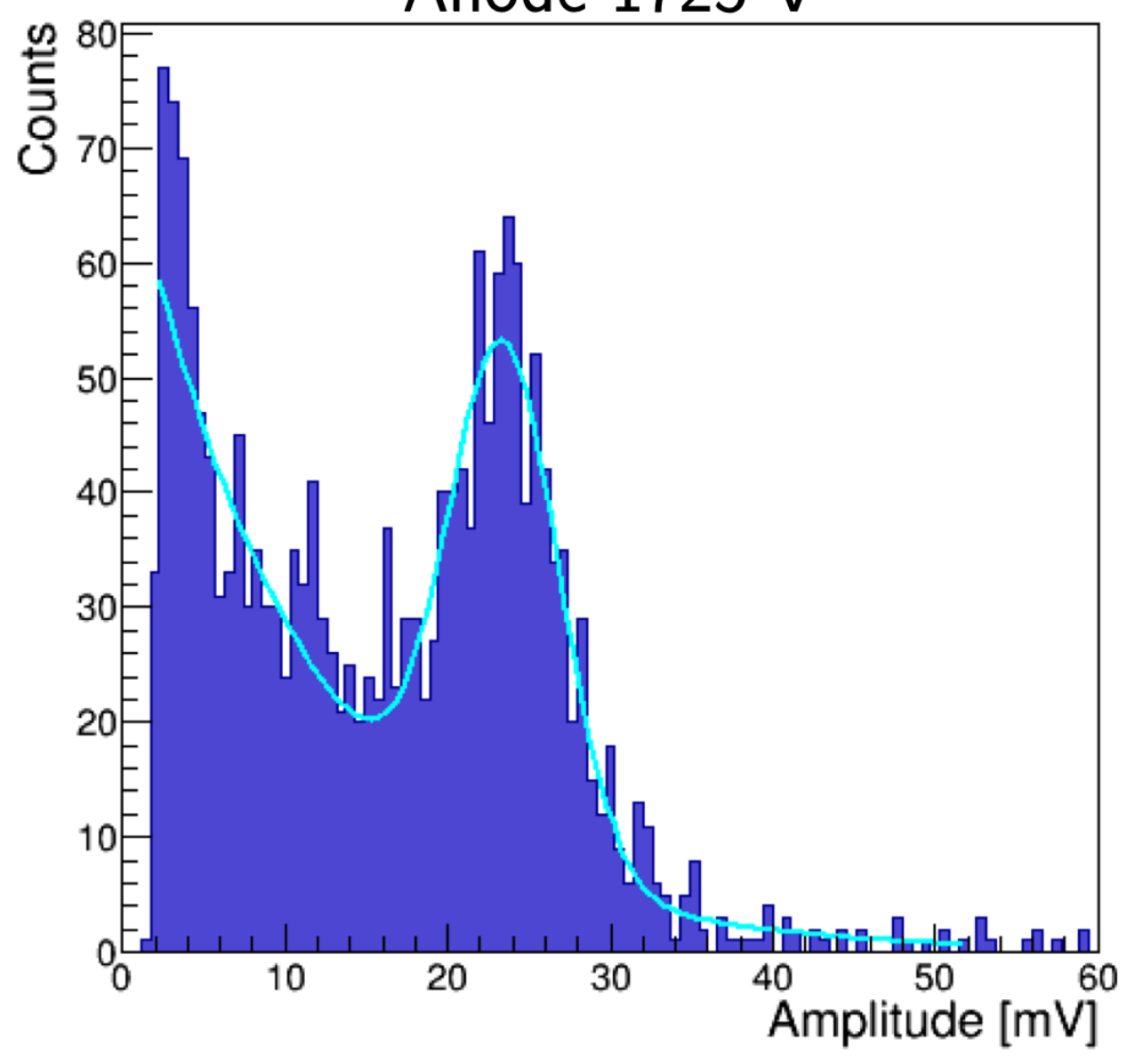

Anode $1750 \mathrm{~V}$

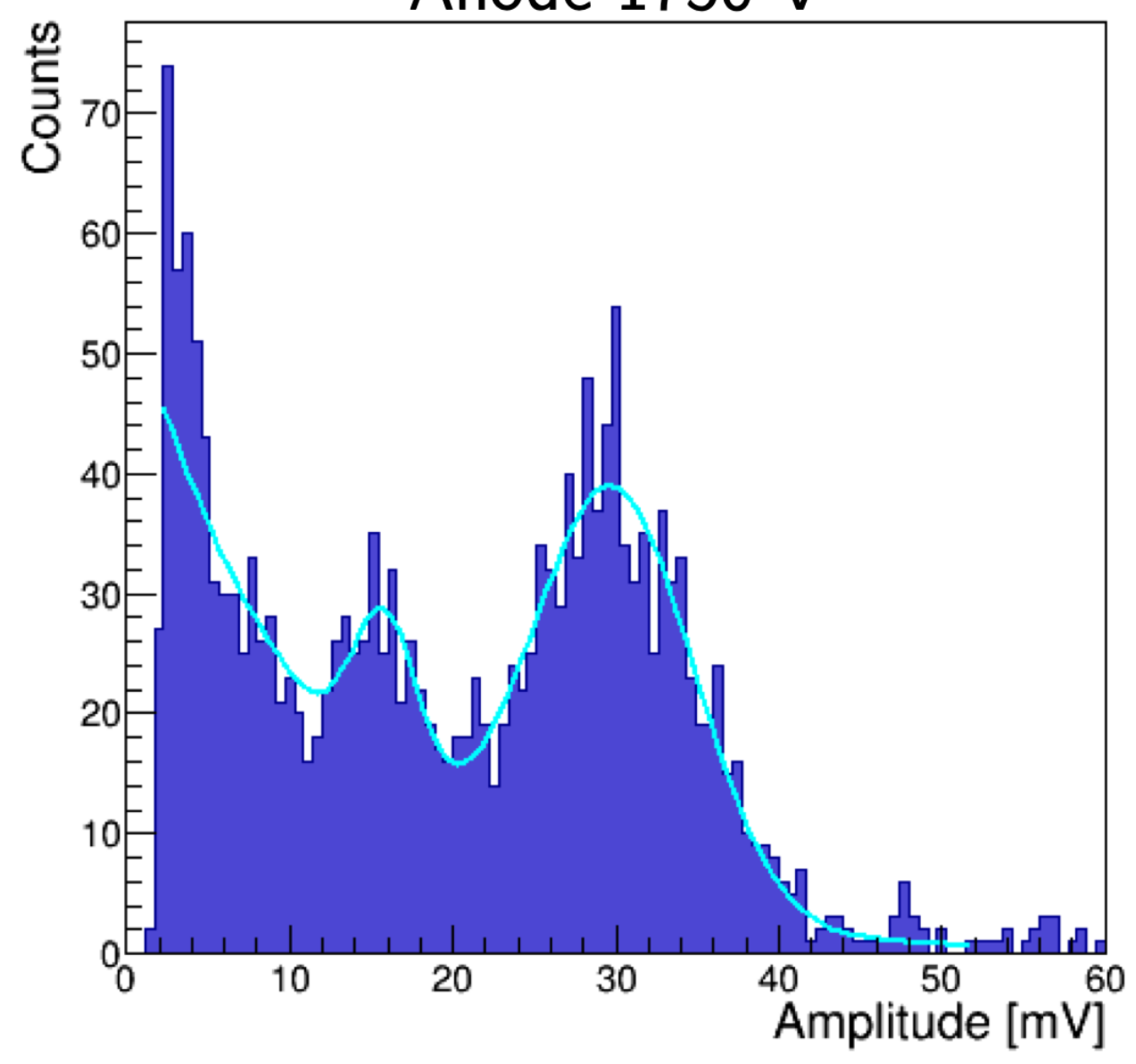

- Typical amplitude spectrum of Fe-55 is observed

- As expected, at fixed pressure, when anode voltage is higher, the peak positions shift to higher amplitudes 


\section{Gain at $1 \mathrm{~atm} \mathrm{Ar}-\mathrm{CO}_{2}$ 90:10}

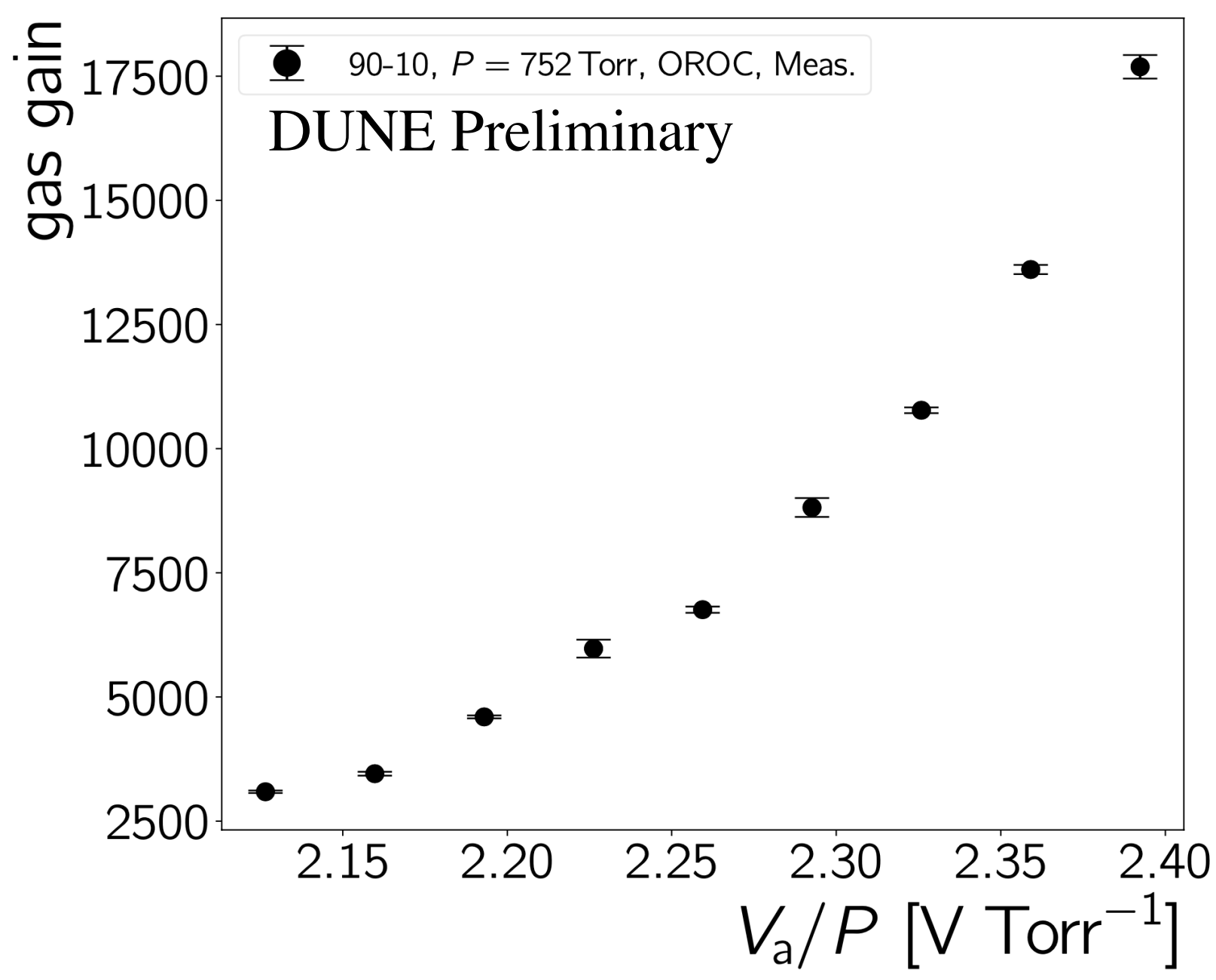

- The expected trend is present: for fixed pressure, a higher anode voltage results in higher gain 


\section{Higher Pressure Operation}

Peak Amplitude Per Trigger for a Fixed Anode Voltage

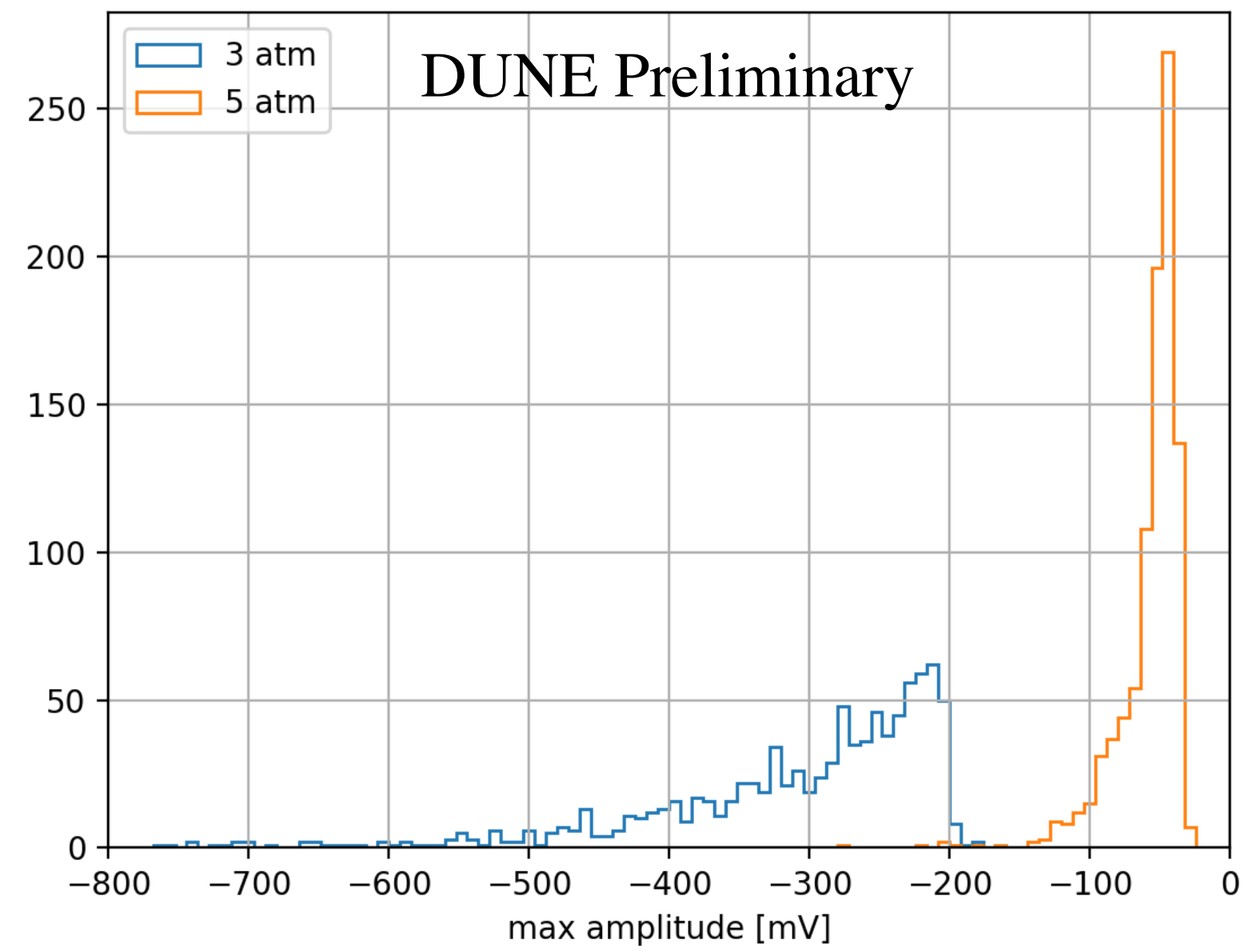

- @ fixed 1.9kV anode voltage, as pressure $\uparrow$, the peak amplitude (as expected) goes down

- Stay tuned for gain at $>1$ atm! 


\section{Summary}

- The HPgTPC is a crucial component of the near detector suite:

$\star$ Extends neutrino cross section measurements to lower energies in region where data are sparse and neutrino interaction models disagree

- We have test stands that primarily test the ALICE's inner and outer readout chambers as part of the on-going R\&D efforts towards building a HPgTPC:

$\star$ In both test stands, we have calibrated the gain at $1 \mathrm{~atm}$ and we observe the expected trend

$\star$ We are also operating these readout chambers at high pressures; stay tuned for gain calibration at $>1 \mathrm{~atm}$

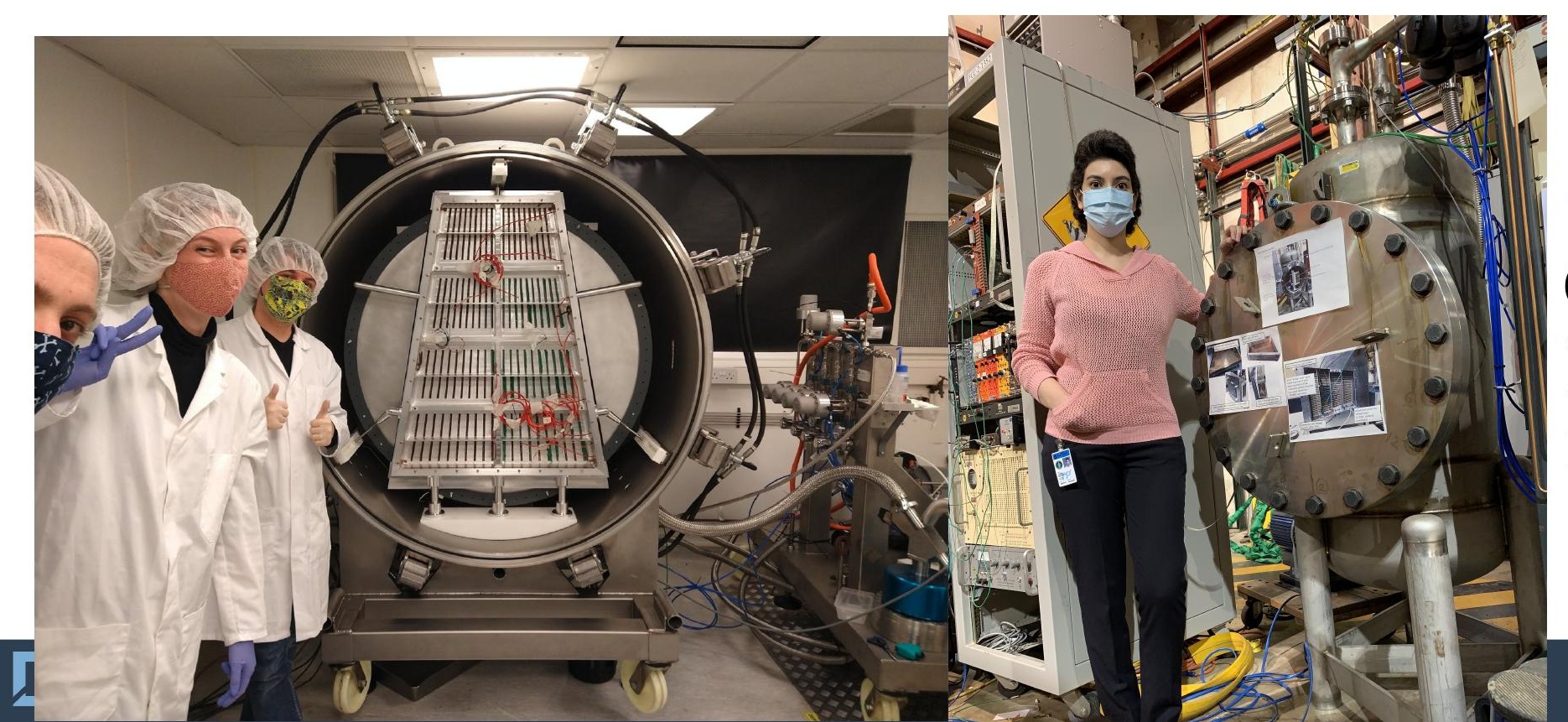

Thank you!

Questions are welcome, here or via email: mtanaz@fnal.gov 


\section{Additional Slides}

